\title{
ANÁLISE DE ESTILOS COGNITIVOS DE GESTORES APLICADOS A UM EMPREENDIMENTO DE ENGENHARIA
}

Paulo Roberto Maciel ${ }^{1}$

Jefferson Lopes La Falce ${ }^{2}$

Cristiana Fernandes De Muylder ${ }^{2}$

Alexandre Teixeira Dias ${ }^{2}$

${ }^{1}$ PROGRAMA DE DOUTORADO E MESTRADO EM ADMINISTRAÇÃO / FACE/FUMEC / UNIVERSIDADE FUMEC

${ }^{2}$ UNIVERSIDADE FUMEC 


\section{ANÁLISE DE ESTILOS COGNITIVOS DE GESTORES APLICADOS A UM EMPREENDIMENTO DE ENGENHARIA}

Resumo: Este artigo se propõe a avaliar o estilo cognitivo individual de gerentes e gestores de empresas distintas, que compuseram um time de engenharia para realizar um empreendimento de engenharia. Para tal, utilizou-se a escala REI (Rational - Experiential Inventory) (PACINI; EPSTEIN, 1999) - questionário que estabelece a preferência cognitiva individual entre racional e intuitivo. O objeto de análise atende aos apelos da academia para disseminação da escala REI, pela sua robustez teórica e empírica, e ao mesmo tempo atingir uma área pouco explorada, a área de gerenciamento de projetos. Os resultados identificaram estilos cognitivos tanto racionais quanto intuitivos, como recomendam as pesquisas empíricas, isto é, formarem equipes versáteis. A conclusão dessa pesquisa se alinha aos estudos que demostraram ser útil conhecer o estilo cognitivo individual, pois permite adequar equipes de acordo com a tarefa a ser executada, o que implica em melhores tomadas de decisões para as organizações.

Palavras-chave: Tomada de Decisão. Gerenciamento de Projetos. Intuição. REI. 


\section{Introdução}

As decisões do dia a dia do ser humano passam pela interação de dois sistemas ou modos de processamento cognitivo (CHAIKEN; TROPE, 1999; EPSTEIN, 1994; KAHNEMAN; FREDERICK, 2002; SLOMAN, 1996; STANOVICH, 1999), seja na relação pessoal, finanças, educação e local de trabalho (FLETCHER; MARKS; HINE, 2012). Esses modos do pensamento humano são conhecidos por diversas nomenclaturas ou propriedades como: Sistema 1 e Sistema 2, sistemas associativo e baseado em regra, processamentos heurístico e analítico, sistemas experiencial e racional, cognições intuitiva e analítica (STANOVICH, 1999). Os termos Tipo 1 (intuitivo) e Tipo 2 (refletivo), segundo Evans e Stanovich (2013), são aqueles que deveriam ser utilizados, posto que, o ser humano tem diversos sistemas mentais. Todo esse conjunto de estudos de como pensa o ser humano, compreende o que se chama teorias de processos ou sistemas duais (EVANS, 2008; FRANKISH, 2010).

Uma das características das teorias duais é a comprovação empírica da relação ortogonal dos construtos racional e intuitivo, isto é, embora sejam processos independentes, podem interagir (AKINCI; SADLER-SMITH, 2013; HODGKINSON et al., 2009b; PACINI; EPSTEIN, 1999). Diferentemente, a teoria unitária referente aos mesmos construtos, estabelece que o racional e o intuitivo estão em polos opostos de um mesmo eixo. Contudo resultados de pesquisas empíricas, associadas a esse modelo, mostram inconsistência em relação a base teórica (HODGKINSON; SADLER-SMITH, 2003; HODGKINSON et al., 2009b).

Para testar as teorias que abordam as diferenças do processamento mental humano, foram desenvolvidas escalas individuais de preferências cognitivas. Segundo Armstrong, Cools e Sadler-Smith (2012), Hodgkinson e Clarke (2007), a MBTI (Myers-Briggs Type Indicator) é uma das escalas de medição de estilo de cognição mais difundidas em diversos domínios, como o gerenciamento. Entretanto, vários resultados de pesquisas referenciadas nessa escala, mostraram inconsistências (GARDNER; MARTINKO, 1996; PETTINGER, 2005) ou incompatibilidade com os fundamentos de sua origem - o trabalho de Jung (Hodgkinson \& Clarke, 2007). Outra escala a destacar é a escala CSI (Cognitive Style Index), baseada na teoria unitária (Hodgkinson \& Sadler-Smith, 2003; Hodgkinson et al., 2009b). A escala REI (Rational-Experiential Inventory) (PACINI; EPSTEIN, 1999) fundamentada nas teorias duais, é rival da CSI (AKINCI; SADLER-SMITH, 2013, SADLER-SMITH, 2011) e contemporânea à escala MBTI.

Uma vez conhecido, por meio das escalas, o grau da preferência cognitiva do indivíduo, foi possível subdividir a relação racional - intuitivo em quatro categorias: versátil (racional alto / intuitivo alto), analítico (racional alto / intuitivo baixo), intuitivo (racional baixo / intuitivo alto) e indiferente (racional baixo / intuitivo baixo). Essa categorização / representação está suportada por Akinci e Sadler-Smith (2013) e Hodgkinson e Clarke (2007).

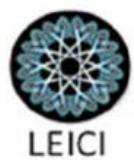


Assim, a escala REI é um instrumento que permite classificar decisores cognitivamente. Diante deste contexto, busca-se aplicar a referida escala de quatro categorias aos gerentes e gestores de equipes de engenharia. O empreendimento de engenharia em questão é do tipo industrial, e o escopo compreendeu o projeto (revisão) e a montagem de uma planta de processamento mineral. Envolveu basicamente as disciplinas de engenharia civil, mecânica, elétrica e automação. Surge daí a pergunta norteadora deste artigo: como está configurado o estilo cognitivo individual de gerentes e gestores de um empreendimento de engenharia localizado no Estado de Minas Gerais?

Do ponto de vista acadêmico é pertinente a pesquisa, pois embora a escala REI tenha robustez teórica e empírica, há carência de estudos, conforme Armstrong, Cools e Sadler-Smith (2012), inclusive no gerenciamento (SADLER-SMITH; BURKE-SMALLEY, 2015). Além disso, as organizações podem se beneficiar ao verificar, se há aderência entre as preferências cognitivas dos gestores e as tarefas a serem executadas, e finalmente concluir, entre outras ações, pela melhoria do processo de tomada de decisões como um todo.

\section{Referencial Teórico}

A evolução dos estudos sobre as teorias duais de processamento cognitivo (CHAIKEN; TROPE, 1999), e seus desdobramentos, como por exemplo, o trabalho sobre heurísticas e vieses (KAHNEMAN; FREDERICK, 2002), reforçaram assertiva de Epstein (1994), ao afirmar que não faltavam evidências para demonstrar que o homem compreende a realidade do dia a dia de duas maneiras fundamentalmente distintas. Uma denominada intuitiva, automática, natural, não verbal, narrativa e experiencial, e a outra analítica, deliberativa, verbal e racional. Sloman (1996), na mesma ordem, denominou o primeiro de sistema associativo, que se aprende por meio da similaridade e contiguidade adquirida na experiência pessoal, e o segundo de sistema baseado em regras, que se aprende pela linguagem, cultura e sistemas formais, ou seja, por manipulação simbólica. Stanovich (1999), em sua revisão sobre os modelos duais, agrupou os termos utilizados pelos teóricos para cada um dos dois sistemas, e os designou respectivamente como Sistema 1, acentuadamente inconsciente, holístico, rápido, sem relativa demanda da capacidade computacional mental, e Sistema 2, tipicamente um processo controlado pela inteligência analítica, lento e com relativa demanda da capacidade mental. A esse conjunto de estudos da forma de pensamento humano, a academia cunhou o termo "teorias de processos ou sistemas duais" (EVANS, 2008; FRANKISH, 2010). Evans e Stanovich (2013), em resposta aos críticos das teorias duais, sugeriram que sejam adotadas as nomenclaturas (processos) Tipo 1 e Tipo 2, porque embora a denominação de Stanovich (1999) - Sistema 1 e Sistema 2 - tenha se tornado popular, a ideia de "dois sistemas" parece ambígua. Segundo Evans e Stanovich (2013), ela pode ser utilizada como sinônimo para a hipótese de duas mentes, mas ir além dos dois tipos de processos. E também transparecer que se refere a um sistema singular, quando na realidade o cérebro tem um conjunto de subsistemas envolvidos no processo Tipo 1, tais como modular, habitual e automático (EVANS, 2008; STANOVICH, 2011).

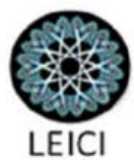


Uma característica comum, identificada nos estudos empíricos das teorias duais, é a relação ortogonal entre o racional e o intuitivo, isto é, eles operam independentemente, mas com possibilidade de interação entre os dois construtos durante o processamento da informação. O trabalho de Epstein et al. (1996), por exemplo, partiu dos pressupostos teóricos estabelecidos pela CEST (Cognitive-Experiential Self-Theory), que são: o paralelismo e a possibilidade de interação entre os construtos racional e intuitivo, inversamente ou de forma ortogonal (ou independente). Ao final da pesquisa, eles confirmaram empiricamente a interação entre os construtos como uma operação independente de relação ortogonal. Pacini e Epstein (1999) revisaram e aprimoraram o estudo de Epstein et al. (1996), e os resultados levaram às mesmas conclusões da pesquisa original, assim como ocorreu com outros trabalhos independentes: Sadler-Smith (2004), Björklund e Bäckström (2008), Hodgkinson et al. (2009b); e Akinci e Sadler-Smith (2013). Contudo as teorias duais têm um modelo tradicionalmente rival, a teoria unitária (AKINCI; SADLER-SMITH, 2013; HODGKINSON; SADLER-SMITH, 2003; SADLER-SMITH, 2011). Essa teoria propõe que a relação racional-intuitivo ocorra unidimensionalmente, o que significa um eixo contínuo com esses dois construtos nos extremos opostos. Porém os resultados empíricos baseados nesse modelo são inconsistentes com o próprio fundamento da teoria unitária, pois os resultados sugerem que os construtos racional e intuitivo se relacionam em mais de uma dimensão e de forma independente (HODGKINSON; SADLER-SMITH, 2003; HODGKINSON et al., 2009b).

A relação entre teoria e construtos, ou a forma de medir o grau dessa relação ocorre por meio de escalas (DEVELLIS, 2017; ZELLER; CARMINES, 1980). No campo das teorias de cognição, uma das escalas mais difundidas é a MBTI (ARMSTRONG; COOLS; SADLER-SMITH, 2012; HODGKINSON; CLARKE, 2007). Essa escala está baseada na teoria multidimensional de Jung, que tipifica a personalidade das pessoas, combinando quatro processos mentais (sensação, intuição, pensamento e sentimento) e duas atitudes (extrovertido e introvertido). A ideia prática dos autores da escala foi estabelecer uma relação entre a personalidade (da pessoa) e o (tipo de) trabalho através de um questionário (MCCAULLEY; MARTIN, 1995). É uma escala aplicada em vários domínios. Segundo Armstrong, Cools e Sadler-Smith (2012), em uma revisão de literatura abrangendo o período de 1969 a 2009 (40 anos), na área de negócios e gerenciamento, o MBTI está presente em sete dos oito temas classificados pelos autores: vocacional e ocupacional, cultura nacional, trabalho em grupo e relações interpessoais, aprendizagem, tomada de decisão, criatividade, inovação e empreendedorismo, vendas e marketing e finalmente sistemas da informação e gerenciamento da informação. Entretanto as análises de Gardner e Martinko (1996) e Pettinger (2005), em estudos que utilizam essa metodologia, apontaram inconsistências nos resultados relativos à teoria de Jung.

Além da escala MBTI, a revisão de literatura de Armstrong, Cools e Sadler-Smith (2012) destacou mais escalas. Os critérios básicos adotados por esses autores para selecionar os artigos e escalas foram: o potencial do artigo e a referência cruzada do autor, assim, dos 4.689 estudos permaneceram 328. Desse total, $24 \%$ dos estudos foram realizados na escala MBTI, $14 \%$ na CSI e 7\% na REI. Foram citadas ainda as escalas KAI (Kirton's AdaptionInnovation Inventory-21\%) e FDI (Field Dependence-Independence-10\%). Contudo, essas

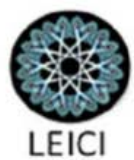


duas últimas abordagens foram descartadas do presente artigo por não serem baseadas na teoria nos construtos racional e intuitivo (ARMSTRONG; COOLS; SADLER-SMITH, 2012). Dessa forma, a seguir, faz-se um paralelo da escala CSI e REI.

A escala CSI tem como construtos a análise e intuição, porém é baseada na teoria unitária, isto é, existe apenas uma dimensão, um eixo contínuo, onde nos polos extremos estão esses dois construtos (HODGKINSON; SADLER-SMITH, 2003; HODGKINSON et al., 2009b). Segundo Cools e Van den Broeck (2007), os polos são tratados frequentemente como dicotômicos e desta forma, conforme Sadler-Smith (2004), impede que a pessoa assuma simultaneamente uma posição fraca ou forte para ambos os polos. Outras críticas vieram de Hodgkinson e Sadler (2003) e Hodgkinson et al. (2009b). Esses estudiosos demonstraram por pesquisa empírica, que a CSI está mais relacionada a uma teoria dual de construtos independentes do que uma teoria unitária-unidimensional como preconiza a teoria onde se baseia.

A escala REI tem como tradicional rival, a escala CSI (AKINCI; SADLER-SMITH, 2013, SADLER, 2011) e está fundamentada na CEST, uma teoria dual de construtos (racional e intuitivo) independentes de relação ortogonal e com interação entre eles (AKINCI; SADLER-SMITH， 2013; HODGKINSON; LANGAN-FOX; SADLER-SMITH， 2008; PACINI; EPSTEIN, 1999). O Rational-Experiential Inventory (REI) é um questionário produzido por Epstein et al., 1996, com 31 assertivas divididas em duas escalas distintas aderente a base teórica dual CEST. Uma delas é a Need for Cognition (NFC) com 19 assertivas com o propósito de capturar o pensamento racional e outra Faith in Intuition (FI) com 12 assertivas para avaliar o grau de confiança nos próprios sentimentos e primeiras impressões como base para as decisões e ações. Posteriormente, Pacini e Epstein (1999) reformularam o questionário para 40 assertivas. Enquanto a escala NFC direcionava a maioria das assertivas para a propensão do indivíduo usar aquele sistema (o racional), a escala FI se concentrava na habilidade para usa-la (a intuição). Assim, um novo questionário procurou corrigir esses aspectos, ao criar as subescalas de propensão e habilidade para cada um dos construtos. Entretanto, os resultados empíricos mostraram aderência das subescalas apenas para a parte racional, ou seja, foi possível correlacionar independentemente propensão e habilidade quando utilizado o pensamento analítico, porém não foi possível diferencia-los para o pensamento experiencial ${ }^{1}$. A despeito desses resultados, Pacini e Epstein (1999) certificaram-se empiricamente da melhora da escala REI para distinção dos construtos racional e experiencial.

De acordo com Akinci e Sadler-Smith (2013) as escalas produzidas pelas teorias duais, relacionadas aos construtos racional-intuitivo, tem aplicações práticas que incluem: a seleção e avaliação, adequação pessoa-organização, adequação pessoa-trabalho, orientação vocacional, composição e construção da equipe e treinamento e desenvolvimento. Isso é possível, segundo os autores, porque as escalas racional-intuitivo nesse modelo são distintas e não trabalham em oposição, e sim simultaneamente, de acordo com a preferência cognitiva ou

${ }^{1}$ Segundo Epstein (2010) o termo experiencial adotado por ele, abrange tanto o conceito intuitivo quanto heurístico.

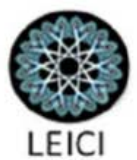


pela adequação à necessidade/tarefa. Por essa característica independente e ortogonal dos construtos racional-intuitivo dos modelos teóricos duais, quando aplicada um instrumento de medição, por exemplo, a escala REI, é possível categorizar o indivíduo conforme Figura 1.

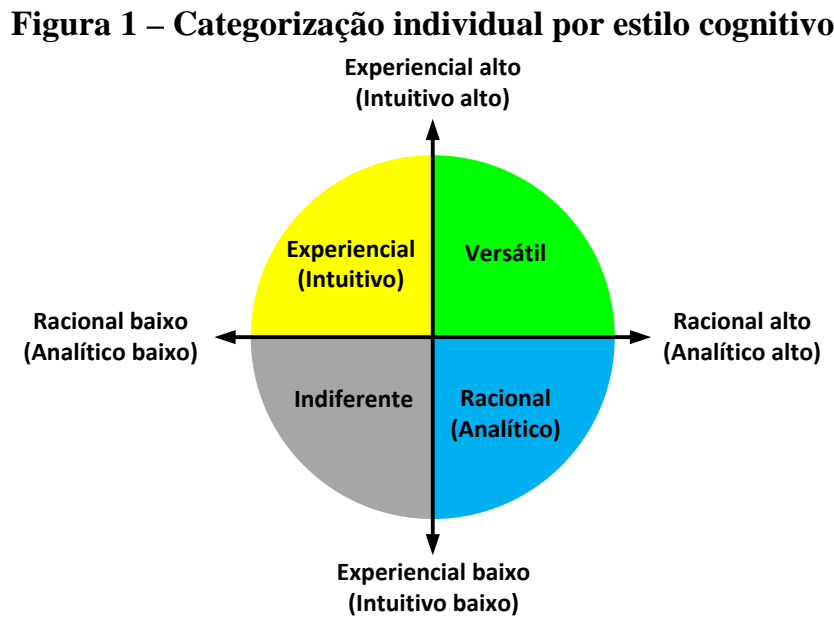

Adaptado pelos autores de Akinci e Sadler-Smith (2013, p. 218) e Hodgkinson e Clarke (2007, p. 246).

As quatro características individuais atribuídas às categorizações da Figura 1, de acordo com Hodgkinson e Clarke (2007), são as seguintes: experiencial (racional baixo / intuitivo alto) é aquela pessoa que se preocupa em enxergar o problema como um todo, mesmo à custa da perda de detalhes, e tem pouca ou nenhuma abordagem analítica. Racional (racional alto / intuitivo baixo) é aquele indivíduo altamente analítico, que se dedica aos detalhes dos dados disponíveis e realiza a decisão passo a passo. Porém se a quantidade de dados é grande e há restrição de tempo, pode enfrentar dificuldade. Além desses aspectos, tem pouca ou nenhuma intuição. Versátil (racional alto / intuitivo alto) é aquela pessoa que pode lidar com detalhes analiticamente, e também ignorar partes, quando necessário. Pode receber tarefas tanto de natureza racional quanto intuitiva. Indiferente (racional baixo / intuitivo baixo) é aquele indivíduo que nem é fortemente racional nem fortemente intuitivo. Não consegue formular uma visão estratégica, pois não consegue extrair detalhes ou visualizar o todo. São pessoas que dependem da opinião e sabedoria de outros, o que alivia o próprio processamento analítico e intuitivo.

A revisão de literatura de Armstrong, Cools e Sadler-Smith (2012) ressaltou a necessidade de aplicação da escala REI, tanto pela sua coerência e consistente base teórica, e também pela sua baixa disseminação. Por exemplo, na área de engenharia, as escalas mais usualmente aplicadas são MBTI, FDI e KAI, de acordo com o trabalho de Armstrong, Cools e Sadler-Smith (2012). Outra revisão de literatura, de Cools, Armstrong e Verbrigghe (2014), concentrada nas áreas de psicologia e gerenciamento, os autores analisaram 234 artigos no período de 1986 a 2010 (25 anos). Eles identificaram as escalas cognitivas aplicadas e recomendaram a utilização, entre outras, da escala REI, tanto para comprovar a evidência da independência dos construtos, como especificamente dar relevância na pesquisa no campo do 
gerenciamento, ao aproximar das ações do cotidiano de trabalho. Nesse sentido, Sadler-Smith e Burke-Smalley (2015) afirmaram que o último trabalho de pesquisa de referência sobre estilos cognitivos na área de gerenciamento foi de Agor (1986).

A seguir serão abordados os pressupostos metodológicos do artigo.

\section{Metodologia}

A pesquisa adotada neste trabalho se configura como descritiva, por identificar a prevalência de um fenômeno (YIN, 2014), transversal pela obtenção dos dados de pesquisa em momento único em relação às etapas de um empreendimento e com abordagem quantitativa (BRYMAN; BELL, 2015).

Inicialmente é necessário contextualizar o ambiente e os atores envolvidos na definição do objeto de análise. Aderente às solicitações de Armstrong, Cools e Sadler-Smith (2012), Cools, Armstrong e Verbrigghe (2014) e Sadler-Smith e Burke-Smalley (2015) foi escolhido um grupo de gestores envolvidos em um empreendimento de engenharia. O empreendimento em questão refere-se a um conjunto de atividades de período definido, isto é, temporário. Normalmente nesses casos, as empresas contratadas pelo cliente final compõem uma força-tarefa ou um time para a execução daquele determinado empreendimento, também com caráter temporário. Os colaboradores das empresas são escolhidos conforme disponibilidade e compatibilidade com as tarefas, e se necessário, contratados novos.

Especificamente nesse empreendimento de engenharia estavam envolvidas oito empresas: as projetistas (3), as fiscalizadoras (2), a fornecedora (1), a construtora (1) e o cliente final (1). As relações entre elas eram as seguintes (Figura 2): A empresa "A", além de ter sido a responsável pela fiscalização geral de construção, também foi coordenadora / fiscalizadora, via empresa "B", do projeto de engenharia desenvolvido pelas empresas " $C$ ", "D" e "E". A empresa "F" foi responsável pela construção e a empresa "G", o cliente final. A empresa "H" atuou como fornecedor de um software de integração, isto é, para que todo o sistema documental de engenharia produzido, tramitasse entre os atores de forma sistêmica e controlada, foi adquirido pela empresa "A" da empresa "H", um software de gestão de documentação de engenharia.

Os atores de P01 a P15, da Figura 2, são os representantes das respectivas empresas, que estiveram envolvidos ou na fase de aquisição, ou de desenvolvimento, ou de testes ou mesmo como usuários do software, durante toda a sua utilização no período de construção do empreendimento. Eles foram escolhidos primeiramente, porque estiveram diretamente ligados ou dependentes do software e pela função que exerciam, pois poderiam interferir no andamento e resultado do empreendimento. Assim sendo, temos os seguintes participantes por nível hierárquico: quatro da baixa gerência (BGE), três da média gerência (MGE), oito da alta gerência (AGE), totalizando quinze participantes escolhidos por critério de conveniência e conhecimento dos representantes-chave pelo pesquisador (YIN, 2014), em função da aglutinação natural de (oito) empresas que formaram um grupo para realizar um

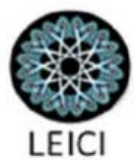


empreendimento comum. Para Bryman e Bell (2015) representa uma oportunidade que poderia ser perdida, pelo próprio propósito temporário de formação do grupo, além disso, como alertou Sadler-Smith e Burke-Smalley (2015), há necessidade de pesquisas no campo de trabalho, efetivamente, por exemplo, para entender melhor o emprego da intuição

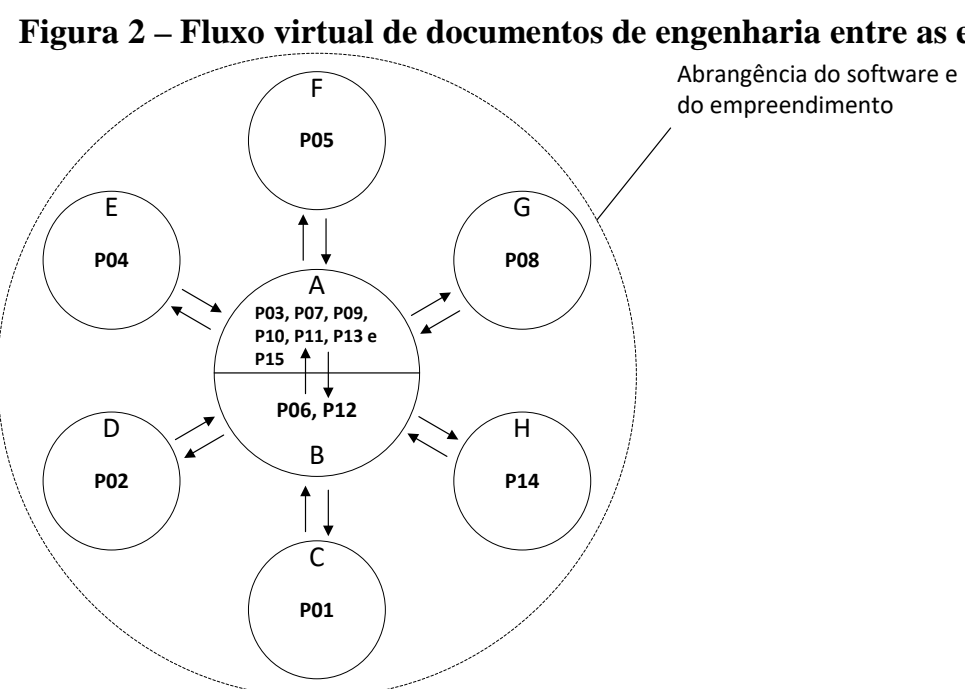

Fonte: Elaborado pelos autores baseado no ambiente pesquisado.

Foi utilizado o questionário REI traduzido de Pacini e Epstein (1999) para a língua portuguesa. Ele contém quarenta assertivas divididas em duas subescalas. Vinte assertivas são relativas à subescala racional (NFC) e vinte assertivas referem-se à subescala intuitiva. Cada item do questionário deve ser respondido pelo participante em uma escala Likert de cinco pontos discretos, ou seja, 1, 2, 3, 4 e 5; sendo que, o valor 1 corresponde ao "completamente falso" e o valor 5 ao "completamente verdadeiro".

O questionário foi aplicado a cada um dos participantes P01 a P15 em momentos distintos, sem tempo pré-determinado para o total preenchimento, porém devendo ser preenchido em apenas um período. Nenhum dos participantes teve acesso prévio ao conteúdo e foram orientados a não comentar sobre o conteúdo com terceiros após a tarefa cumprida. Além disso, não tiveram conhecimento quem foram os demais pares escolhidos.

Uma vez computados os índices para as partes racional e intuitiva independentemente, conforme Pacini e Epstein (1999), foi adotado a análise de correspondência entre os construtos (HAIR et al. 2014). De acordo com esses autores, análise de correspondência consiste da tabulação cruzada entre as dimensões a serem analisadas, que pode ser representado espacialmente por meio de um mapa perceptual, como teorizado nos trabalhos de Akinci e Sadler-Smith (2013) e Hodgkinson e Clarke (2007).

\section{Resultados e análise}


Uma vez preenchidos os questionários, por cada um dos quinze participantes, as respostas foram computadas, conforme a escala adota (Tabela 1).

Tabela 1 - Pontuação do questionário REI e Z-score

\begin{tabular}{llllll}
\hline & \multicolumn{2}{c}{ Estilo Cognitivo } & & \multicolumn{2}{c}{$Z-$ Score } \\
\cline { 2 - 3 } \cline { 5 - 6 } Participante & Racional & Intuitivo & & Racional & Intuitivo \\
\hline P01 & 3,50 & 2,95 & & $-1,85$ & $-0,38$ \\
P02 & 4,00 & 4,15 & & $-0,16$ & $+1,76$ \\
P03 & 4,30 & 2,70 & & $+0,86$ & $-0,83$ \\
P04 & 4,10 & 2,85 & & $+0,18$ & $-0,56$ \\
P05 & 4,00 & 3,10 & & $-0,16$ & $-0,11$ \\
P06 & 4,60 & 3,00 & & $+1,87$ & $-0,29$ \\
P07 & 3,65 & 3,05 & & $-1,34$ & $-0,20$ \\
P08 & 4,20 & 3,60 & & $+0,52$ & $+0,78$ \\
P09 & 4,40 & 2,25 & & $+1,19$ & $-1,63$ \\
P10 & 3,75 & 3,70 & & $-1,00$ & $+0,96$ \\
P11 & 4,05 & 4,25 & & $+0,01$ & $+1,94$ \\
P12 & 3,70 & 3,30 & & $-1,17$ & $+0,24$ \\
P13 & 4,15 & 2,60 & & $+0,35$ & $-1,01$ \\
P14 & 4,20 & 2,75 & & $+0,52$ & $-0,74$ \\
P15 & 4,10 & 3,20 & $+0,18$ & $+0,07$ \\
\hline Média & $\mathbf{4 , 0 5}$ & $\mathbf{3 , 1 6}$ & & \\
\hline Desvio Padrão & $\mathbf{0 , 3 0}$ & $\mathbf{0 , 5 6}$ & & & \\
\hline
\end{tabular}

Fonte: Elaborado pelos autores.

Segundo Carley e Frantz (2009), as organizações não tomam decisão e sim os indivíduos em nome delas. Por essa razão, segundo esses autores, a tomada de decisão organizacional está pautada na decisão individual. Portanto se as empresas de engenharia ou as pessoas que a compõem, pela própria natureza do trabalho, tendem a racionalizar a sua atividade (GAREL, 2013; SHENHAV, 2003), é mais provável que esses integrantes sejam mais racionais do que intuitivos. Os dados corroboram tal inferência, pois dos quinze participantes, apenas P02 e P11 são mais intuitivos do que racionais, ou seja, aproximadamente $90 \%$ dos participantes dessa amostragem são mais racionais.

Como destacado por Fletcher, Marks, e Hine (2012), que também utilizaram a escala REI, a coluna Z-Score permite que se verifique dentro da própria amostragem, o quanto mais ou menos racionais ou intuitivos entre os participantes daquele grupo. Por conseguinte, é possível estabelecer as categorizações conforme Akinci e Sadler-Smith (2013) e Hodgkinson e Clarke (2007) (Figura 3). É possível dizer que dentro da categoria versátil, os três participantes estão na alta gerência (AGE). Na categoria racional há três na AGE, um na média gerência (MGE) e dois na baixa gerência (BGE). Na categoria experiencial há um na AGE, outro na MGE e outro na BGE. E finalmente na categoria indiferente repete-se a mesma distribuição da categoria experiencial.

Figura 3 - Mapa do questionário REI e Z-score 


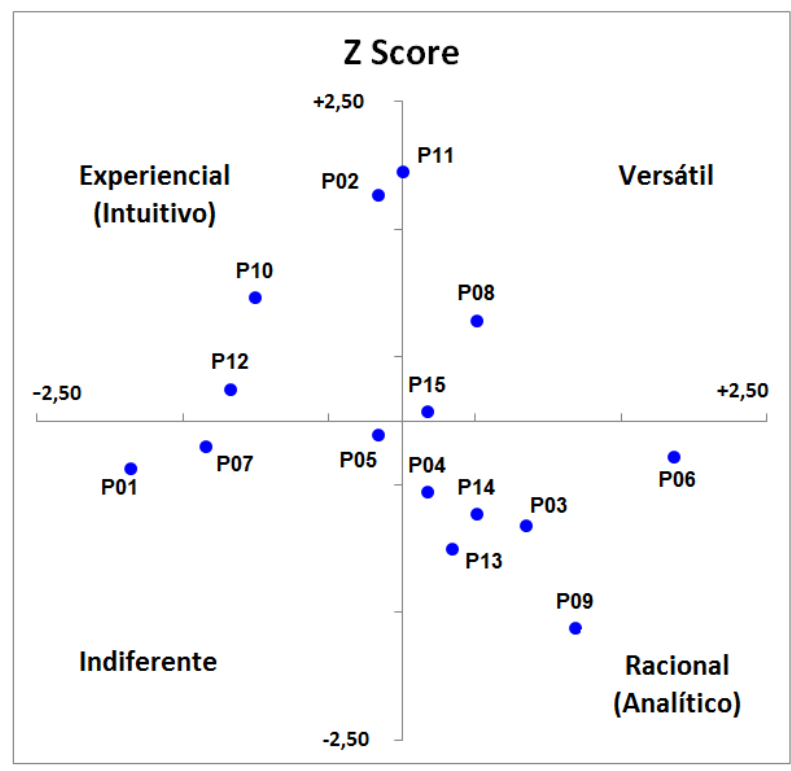

Fonte: Elaborado pelos autores.

Os dados verificados na tabela 1 mostram que há mais gestores racionais do que intuitivos, o mesmo ocorreu pela categorização de Akinci e Sadler-Smith (2013) e Hodgkinson e Clarke (2007), apresentada na Tabela 2. A amostra desse estudo mostra que a categoria racional (40\%) é o dobro de qualquer das outras três (20\%) categorias. Assim, os resultados espelhados nas tabelas 1 e 2 parecem indicar que, ser mais racional é o perfil ideal ou necessário às pessoas que integram as empresas ou times de engenharia. Porém, de acordo Böhle, Heidling e Schoper (2016), projetos são essencialmente limitados em tempo assim como uma fonte de incertezas, e nessas circunstâncias a utilização da intuição para as decisões tem melhor desempenho do que o pensamento analítico conforme pesquisas empíricas.

Tabela 2 - Categorização cognitiva por função

\begin{tabular}{|c|c|c|c|c|c|}
\hline \multirow[b]{2}{*}{ Categoria } & \multicolumn{3}{|c|}{ Nível hierárquico } & \multirow[b]{2}{*}{ Total } & \multirow[b]{2}{*}{$\%$} \\
\hline & AGE & MGE & BGE & & \\
\hline Versátil & 3 & 0 & 0 & 3 & 20 \\
\hline Racional & 3 & 1 & 2 & 6 & 40 \\
\hline Experiencial & 1 & 1 & 1 & 3 & 20 \\
\hline Indiferente & 1 & 1 & 1 & 3 & 20 \\
\hline Total & 8 & 3 & 4 & 15 & 100 \\
\hline$\%$ & 53 & 20 & 27 & 100 & \\
\hline
\end{tabular}


Esse resultado foi também indicado no trabalho de Agor (1986) que comprovou após testar mais de dois mil gestores de diversas áreas. A pesquisa de Burke e Miller (1999) em áreas assemelhadas também chegou à mesma conclusão, da mesma forma que Leybourne e Sadler-Smith (2006), Dane e Pratt (2007, 2009), Hodgkinson et al. (2009a), Dayan e Di Benedetto (2011) e Elbanna (2015).

A Tabela 2 ainda mostra que, 53\% da amostra desse estudo encontra-se na AGE. Isso indica uma representação significativa daqueles atores envolvidos nas decisões de última instância, que se pressupõem as estratégicas. Os demais agentes (47\%), subdivididos na média e baixa gerência são tão necessários quanto, pois segundo Floyd e Wooldridge (1997) e Wooldridge, Schmid e Floyd (2008), esses gerentes e gestores podem influenciar as decisões estratégicas e mudanças organizacionais no sentido de torna-las efetivas ou não.

Assim como o modo analítico, a intuição também está presente como estilo cognitivo em todos os participantes dessa amostra. Contudo, seis participantes (P02, P08, P10, P11, P12, P15) ou 40\% da amostra estão acima da média em intuição, segundo a coluna Z-Score da Tabela 1 (ou Figura 3). Ao fazer a correspondência desse grupo com a Figura 2, nota-se que 50\% deles (P10, P11 e P15) são colaboradores da empresa "A”, aquela responsável pela catalisação, coordenação e gerenciamento de todas as ações para o empreendimento se realizar. É um indício que a empresa-chave estava preparada para lidar com os tipos de problemas inerentes à situação - limitação de tempo e incertezas (ARTINGER et al., 2015; SALAS; ROSEN; DIAZGRANADOS, 2010).

Três questões emergem, quando comparado os participantes entre categorias, conforme Figura 3. A primeira, P08, P11 e P15, os versáteis são aqueles que pontuaram acima da média tanto em racionalidade como intuição. A segunda, P01, P05 e P07, que fazem parte da categoria indiferente, é o oposto da categoria versátil em pontuação, ou seja, estão abaixo da média em racionalidade e intuição. A terceira, o grupo (P02, P06, P08, P10, P11, P15) são aqueles que a soma linear ou vetorial dos graus de racionalidade e intuição estão acima da média. Nesse grupo, exceto P08, P11 e P15 (os versáteis), aparecem dois dos três mais intuitivos (P02, P10), e somente um dos seis mais racionais (P06). Essa constatação sugere que, para atingir a versatilidade, o caminho inicia-se pela tendência do indivíduo ser mais intuitivo do que mais racional. Evidências parecem apontar nesse sentido. Para Klein (2004) os gestores utilizam a intuição noventa por cento das vezes para lidar em situações difíceis ou até mais para aquelas rotineiras. Esse autor também preconiza que essa utilização depende do acumulo de experiência ou tempo. Porém isso gera um aparente paradoxo em relação ao aprendizado formalizado, isto é, o indivíduo poderia absorver mais rapidamente pelo conhecimento explícito de normas, guias, manuais, procedimentos. Contudo, de acordo com Pretz (2008), o ser humano no dia a dia assimila na prática, mais pelo aprendizado da experiência informal do que aquele formal, deliberado. E essa é a mesma conclusão de Ramazani e Jergeas (2015) e Savelsbergh, Havermans e Storm (2016), especificamente na área de gerenciamento de projetos.

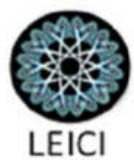




\section{Considerações finais e conclusão}

Este artigo se propôs a avaliar o estilo cognitivo individual de gerentes e gestores de empresas distintas que compuseram um time de engenharia para realizar um empreendimento. Para tal utilizou a escala REI (Rational-Experiential Inventory) - questionário que estabelece a preferência cognitiva individual entre racional e intuitivo. Os resultados apontaram inicialmente para gerentes e gestores de engenharia mais racionais, porém ao avalia-los pela categorização de estilos cognitivos, a equipe de engenharia, nesse estudo de caso, mostrou-se um mix entre racional e intuitivo, como recomendam as pesquisas empíricas, isto é, equipes versáteis. Assim, conhecer o estilo cognitivo individual ou modo de pensamento individual permite adequar equipe de acordo com a tarefa a ser executada, o que implica em melhores tomadas de decisões para as organizações.

Desde Agor (1986) a Savelsbergh, Havermans e Storm (2016) há uma demonstração da superioridade da intuição sobre o pensamento analítico, sob determinadas condições, entretanto, outra conclusão desses trabalhos é que a natureza dos diversos tipos de tomadas de decisão, que permeiam o mundo real, das simples às estratégicas, exigirá dos decisores uma transição de estilos cognitivos (do racional para intuitivo e vice-versa), ou que os grupos de decisão tenham um mix desses estilos de cognição, enfim, que os decisores ou o grupo de decisão "sejam versáteis". Em suma, seja do ponto de vista individual ou de grupo, para conseguir decisões efetivas, o objetivo é escolher indivíduos ou compor grupos, tanto quanto possível, que se enquadrem na categoria versátil.

Basicamente esse artigo tem duas limitações: é um único caso e o tamanho da amostra em si. Sendo que, a segunda limitação está intrinsicamente relacionada à primeira em função do caso disponibilizado e analisado. Assim sendo, futuras pesquisas poderiam diminuir ou eliminar essas fragilidades, se múltiplos casos puderem ser avaliados com a mesma metodologia. O tamanho da amostragem aumentaria em função da própria análise de múltiplos projetos.

Pesquisas futuras também poderiam incorporar a avaliação dos gerentes e gestores de engenharia sobre quais fatores estão envolvidos nas tomadas de decisão durante os empreendimentos, qual a visão e impacto da racionalidade e intuição nessas tomadas de decisão, assim como das incertezas e riscos. Assim como correlacionar a composição dos estilos de cognição com os resultados de seus empreendimentos.

\section{Referências}

AGOR, W. H. The logic of intuition: How top executives make important decisions. Organizational Dynamics, v. 14, n. 3, p. 5-18, 1986.

AKINCI, C.; SADLER-SMITH, E. Assessing individual differences in experiential (intuitive) and rational (analytical) cognitive styles. International Journal of Selection and Assessment, v. 21, n. 2, p. 211-221, 2013.

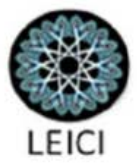


ARMSTRONG, S. J.; COOLS, E.; SADLER-SMITH, E. Role of cognitive styles in business and management: Reviewing 40 years of research. International Journal of Management Reviews, v. 14, n. 3, p. 238-262, 2012.

ARTINGER, F.; PETERSEN, M.; GIGERENZER, G.; WEIBLER, J. Heuristics as adaptive decision strategies in management. Journal of Organizational Behavior, v. 36, n. S1, p. S33-S52, 2015.

BJÖRKLUND, F.; BÄCKSTRÖM, M. Individual differences in processing styles: validity of the Rational-Experiential Inventory. Scandinavian Journal of Psychology, v. 49, n. 5, p. 439-446, 2008.

BÖHLE, F.; HEIDLING, E.; SCHOPER, Y. A new orientation to deal with uncertainty in projects. International Journal of Project Management, v. 34, n. 7, p. 1384-1392, 2016.

BRYMAN, A.; BELL, E. Business research methods. 4th ed. Oxford: University Press, 2015.

BURKE, L. A.; MILLER, M. K. Taking the mystery out of intuitive decision-making. The Academy of Management Executive, v. 13, n. 4, p. 91-99, 1999.

CARLEY, K. M.; FRANTZ, T. L. Modeling organizational and individual decision making. In: SAGE, Andrew. P.; ROUSE, William. B. (Eds.) Handbook of systems engineering and management. 2nd ed. Hoboken: John Wiley \& Sons, 2009, p. 723-762.

CHAIKEN, S.; TROPE, Y. (Eds.). Dual-process theories in social psychology. New York: Guilford Press, 1999.

COOLS, E.; ARMSTRONG, S. J.; VERBRIGGHE, J. Methodological practices in cognitive style research: Insights and recommendations from the field of business and psychology. European Journal of Work and Organizational Psychology, v. 23, n. 4, p. 627-641, 2014.

COOLS, E.; VAN DEN BROECK, H. Development and validation of the Cognitive Style Indicator. The Journal of Psychology, v. 141, n. 4, p. 359-387, 2007.

DANE, E.; PRATT, M. G. Exploring intuition and its role in managerial decision making. Academy of Management Review, v. 32, n. 1, p. 33-54, 2007.

DANE, E.; PRATT, M. G. Conceptualizing and measuring intuition: A review of recent trends. In: HODGKINSON, G. P.; FORD, J. K. (Eds.) International review of industrial and organizational psychology. Chichester: Wiley-Blackwell, v. 24, p. 1-40, 2009. 
DAYAN, M.; DI BENEDETTO, C. A. Team intuition as a continuum construct and new product creativity: The role of environmental turbulence, team experience, and stress. Research Policy, v. 40, n. 2, p. 276-286, 2011.

DEVELLIS, R. F. Scale development: Theory and applications. 4th ed. London: Sage publications, 2017. (Applied social research methods series, v. 26).

ELBANNA, S. Intuition in project management and missing links: Analyzing the predicating effects of environment and the mediating role of reflexivity. International Journal of Project Management, v. 33, n. 6, p. 1236-1248, 2015.

EPSTEIN, S. Integration of the cognitive and the psychodynamic unconscious. American Psychologist, v. 49, n. 8, p. 709-724, 1994.

EPSTEIN, S. Demystifying intuition: What it is, what it does, and how it does it. Psychological Inquiry, v. 21, n. 4, p. 295-312, 2010.

EPSTEIN, S.; PACINI, R.; DENES-RAJ, V.; HEIER, H. Individual differences in intuitiveexperiential and analytical-rational thinking styles. Journal of Personality and Social Psychology, v. 71, n. 2, p. 390-405, 1996.

EVANS, J. St. B. T. Dual-processing accounts of reasoning, judgment, and social cognition. Annual Review Psychology, v. 59, p. 255-278, 2008.

EVANS, J. St. B. T.; STANOVICH, K. E. Dual-process theories of higher cognition: Advancing the debate. Perspectives on Psychological Science, v. 8, n. 3, p. 223-241, 2013.

FLETCHER, J. M.; MARKS, A. D. G.; HINE, D. W. Latent profile analysis of working memory capacity and thinking styles in adults and adolescents. Journal of Research in Personality, v. 46, n. 1, p. 40-48, 2012.

FLOYD, S. W.; WOOLDRIDGE, B. Middle management's strategic influence and organizational performance. Journal of Management Studies, v. 34, n. 3, p. 465-485, 1997.

FRANKISH, K. Dual-process and dual-system theories of reasoning. Philosophy Compass, v. 5, n. 10, p. 914-926, 2010.

GARDNER, W. L.; MARTINKO, M. J. Using the Myers-Briggs Type Indicator to study managers: A literature review. Journal of Management, v. 22, n. 1, p. 45-83, 1996.

GAREL, G. A history of project management models: From pre-models to the standard models. International Journal of Project Management, v. 31, n. 5, p. 663-669, 2013. 
HAIR JR., J. F.; BLACK, W. C.; BABIN, B. J.; ANDERSON, R. E. Multivariate data analysis. 7th ed. Harlow: Pearson Education Limited, 2014.

HODGKINSON, G. P.; CLARKE, I. Conceptual note: Exploring the cognitive significance of organizational strategizing: A dual-process framework and research agenda. Human Relations, v. 60, n. 1, p. 243-255, 2007.

HODGKINSON, G. P.; LANGAN-FOX, J.; SADLER-SMITH, E. Intuition: A fundamental bridging construct in the behavioural sciences. British Journal of Psychology, v. 99, n. 1, p. 1-27, 2008.

HODGKINSON, G. P.; SADLER-SMITH, E. Complex or unitary? A critique and empirical re-assessment of the Allinson-Hayes Cognitive Style Index. Journal of Occupational and Organizational Psychology, v. 76, n. 2, p. 243-268, 2003.

HODGKINSON, G. P.; SADLER-SMITH, E.; BURKE, L. A.; CLAXTON, G.; SPARROW, P. R. Intuition in organizations: Implications for strategic management. Long Range Planning, v. 42, n. 3, p. 277-297, 2009a.

HODGKINSON, G. P.; SADLER-SMITH, E.; SINCLAIR, M.; ASHKANASY, N. M. More than meets the eye? Intuition and analysis revisited. Personality and Individual Differences, v. 47, n. 4, p. 342-346, 2009b.

KAHNEMAN, D.; FREDERICK, S. Representativeness revisited: Attribute substitution in intuitive judgment. In: GILOVICH, T.; GRIFFIN, D.; KAHNEMAN, D. (Eds.) Heuristics and biases: The psychology of intuitive judgment. New York: Cambridge University Press, 2002. p. 49-81.

KLEIN, G. The power of intuition: How to use your gut feelings to make better decisions at work. New York: Currency-Doubleday, 2004.

LEYBOURNE, S.; SADLER-SMITH, E. The role of intuition and improvisation in project management. International Journal of Project Management, v. 24, n. 6, p. 483-492, 2006.

MCCAULlEY, M. H.; MARTIN, C. R. Career assessment and the Myers-Briggs type indicator. Journal of Career Assessment, v. 3, n. 2, p. 219-239, 1995.

PACINI, R.; EPSTEIN, S. The relation of rational and experiential information processing styles to personality, basic beliefs, and the ratio-bias phenomenon. Journal of Personality and Social Psychology, v. 76, n. 6, p. 972-987, 1999.

PRETZ, J. E. Intuition versus analysis: Strategy and experience in complex everyday problem solving. Memory \& Cognition, v. 36, n. 3, p. 554-566, 2008. 
RAMAZANI, J.; JERGEAS, G. Project managers and the journey from good to great: The benefits of investment in project management training and education. International Journal of Project Management, v. 33, n. 1, p. 41-52, 2015.

SADLER-SMITH, E. Cognitive style and the management of small and medium-sized enterprises. Organization Studies, v. 25, n. 2, p. 155-181, 2004.

SADLER-SMITH, E. The intuitive style: Relationships with local/global and verbal/visual styles, gender, and superstitious reasoning. Learning and Individual Differences, v. 21, n. 3, p. 263-270, 2011.

SADLER-SMITH, E.; BURKE-SMALLEY, L. A. What do we really understand about how managers make important decisions? Organizational Dynamics, v. 44, n. 1, p. 9-16, 2015.

SALAS, E.; ROSEN, M. A.; DIAZGRANADOS, D. Expertise-based intuition and decision making in organizations. Journal of Management, v. 36, n. 4, p. 941-973, 2010.

SAVELSBERGH, C. M. J. H.; HAVERMANS, L. A.; STORM, P. Development paths of project managers: What and how do project managers learn from their experiences? International Journal of Project Management, v. 34, n. 4, p. 559-569, 2016.

SHENHAV, Y. The historical and epistemological foundations of organization theory: fusing sociological theory with engineering discourse. In: TSOUKAS, Haridimos; KNUDSEN, Christian (Eds.). The Oxford handbook of organization theory. New York: Oxford University Press, 2003. p. 183-209.

SLOMAN, S. A. The empirical case for two systems of reasoning. Psychological Bulletin, v. 119, n. 1, p. 3-22, 1996.

STANOVICH, K. E. Who is rational? Studies of individual differences in reasoning. Mahwah: Lawrence Erlbaum Associates, 1999.

STANOVICH, K. E. Rationality and the reflective mind. New York: Oxford University Press, 2011.

WOOLDRIDGE, B.; SCHMID, T.; FLOYD, S. W. The middle management perspective on strategy process: Contributions, synthesis, and future research. Journal of Management, v. 34, n. 6, p. 1190-1221, 2008.

YIN, R. K. Case study research: Design and methods. 5th ed. Thousand Oaks: Sage Publications, 2014.

ZELLER, R. A.; CARMINES, E. G. Measurement in the social sciences: The link between theory and data. New York: Cambridge University Press, 1980.

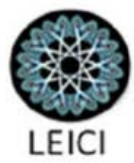

